\title{
A Molecular Dynamics Study on Alumina/Carbon Nanotube Composite: How Does Annealing Affect Mechanical Properties?
}

\author{
Yixin $\mathrm{SU}^{\mathrm{a}}$, Jing ZHANG ${ }^{\mathrm{a}}$, Qian $\mathrm{CHEN}^{\mathrm{a}}$, Yang $\mathrm{WANG}^{\mathrm{a}}{ }^{\mathrm{b}}$, Narumasa MIYAZAKI ${ }^{\mathrm{a}}$, Yusuke OOTANI ${ }^{\mathrm{a}}$, \\ Nobuki OZAWA ${ }^{\text {a }}$, And Momoji KUBO ${ }^{\mathrm{a}^{*}}$
}

${ }^{a}$ Institute for Materials Research, Tohoku University, 2-1-1 Katahira, Aoba-ku, Sendai 980-8577, Japan

${ }^{b}$ Department of Mechanical Systems Engineering, Graduate School of Engineering, Tohoku University, 6-6-01 Aramaki-aza-aoba, Aoba-ku, Sendai 980-8579, Japan

*e-mail:momoji@imr.tohoku.ac.jp

(Received: January 26, 2020; Accepted for publication: March 25, 2020; Online publication: April 25, 2020)

Carbon nanotube (CNT) is conventionally expected to reinforce ceramic matrix like alumina. Previous experiments show that processing conditions such as annealing have positive effect on mechanical properties of the alumina/CNT composite. However, the reinforcing mechanism by annealing is not fully understood yet. For further improving the performance of alumina/CNT composite, this study aims to understand the reinforcing mechanism by annealing from the atomic scale with reactive molecular dynamics simulations. We conduct tensile simulations on pure alumina, non-annealed and annealed alumina/CNT composites, and then analyze the mechanical properties of the three models. Consequently, we find out that annealing enhances the interfacial interactions between alumina and CNT and reduces the interfacial slippage, so that the annealed CNT is more stretched than the non-annealed CNT, finally resulting in an improvement of the mechanical performance of composite.

Keywords: Carbon nanotubes, Ceramic matrix composites, Elastic properties, Reactive molecular dynamics simulations

\section{Introduction}

The combination of carbon nanotube (CNT) and alumina as a composite is expected to exhibit the respective advantages of both components, such as high elastic properties and high hardness. Experimentally, G. Yamamoto et al. has reported that processing conditions like annealing temperature [1] and acid treatment [2] improve the mechanical properties of alumina/CNT composite. However, the reinforcing mechanism by annealing is still unknown. For the further improvement of CNT as reinforcement fiber, we herein use molecular dynamics (MD) simulation approach to reveal the reinforcing mechanism by annealing on the mechanical properties of alumina/CNT composite.

\section{Method and Model}

Firstly, we construct an amorphous alumina model with the cell size of $42 \AA \times 42 \AA \times 125 \AA$, as shown in Figure 1a. Secondly, we dig a hole with the diameter of $17 \AA$ and the length of $96 \AA$ in the amorphous alumina and subsequently a singlewalled CNT with a length of $90 \AA$, a diameter of $13.56 \AA$, and a chiral index of $(10,10)$ is inserted into the hole to form the composite. After $2.5 \mathrm{ps}$ of NPT relaxation (which means total number of atoms, pressure, and temperature are kept unchanged in the system) at $300 \mathrm{~K}$ and $1 \mathrm{~atm}$ by MD simulation to eliminate the internal stress, we obtain a nonannealed composite model with a CNT volume fraction of about $5 \%$, as shown in Figure 1b. Meanwhile, the annealed composite model is constructed by performing MD simulations as following steps under NPT condition: anneal the alumina/ 


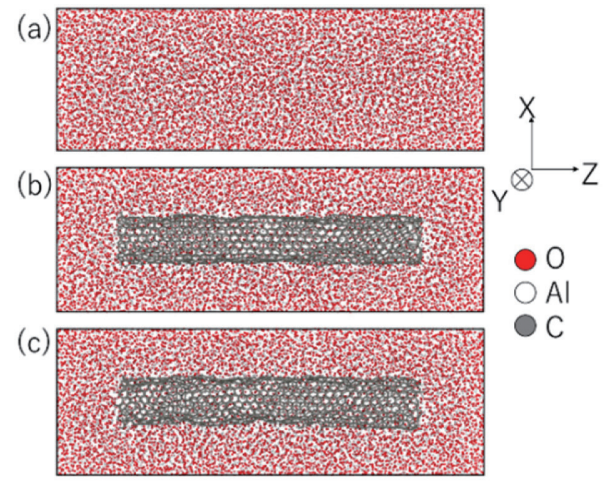

Figure 1. Simulation models of (a) pure amorphous alumina, (b) non-annealed alumina/CNT composite, and (c) annealed alumina/CNT composite.

CNT composite at $1500 \mathrm{~K}$ and $1 \mathrm{GPa}$ for $7.5 \mathrm{ps}$, cool it down to $300 \mathrm{~K}$ at $1 \mathrm{GPa}$ within $2.5 \mathrm{ps}$, and lastly relax it for 2.5 ps at $300 \mathrm{~K}$ and $1 \mathrm{~atm}$ to eliminate the internal stress. All simulations are performed by using Nose-Hoover thermostat. The annealed composite model is shown in Figure 1c. Thus, we succeed to obtain three models: a pure alumina model, a non-annealed composite model, and an annealed composite model.

Tensile simulations are carried out with a strain rate of $1 \mathrm{~ns}^{-1}$ along the $\mathrm{z}$ direction. The simulation temperature is set at $300 \mathrm{~K}$ and a molecular dynamics time step of $0.5 \mathrm{fs}$ is used. Those simulations are performed by our developed MD simulator, LASKYO [3] using the potential of reactive force field (ReaxFF). The parameters for carbon reported by Srinivasan et $a l$. are employed [4], while the parameters for alumina and the interaction between alumina and carbon reported by Hong and Duin are used [5].

\section{Results and Discussion}

To confirm the reinforcing effect by annealing, we investigate the stress-strain curve of the three models (Figure 2). In all curves, stresses increase linearly at first, indicating the elastic deformation regime; whereas, after the strain of around 0.05 , linear relation for all three models disappears, indicating the plastic deformation regime. Young's modulus is represented by the slope of the stress-strain curve in the elastic regime, while tensile strength corresponds to the highest point in the plastic regime. Young's modulus and tensile strength of the non-annealed composite (104.5

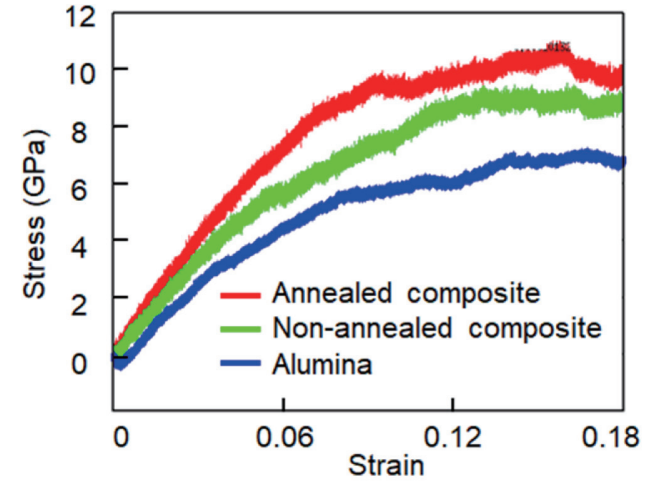

Figure 2. Stress-strain curves for the alumina, non-annealed and annealed composites. (a)

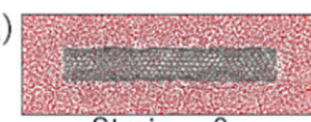
Strain $=0$

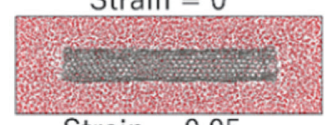

Strain $=0.05$

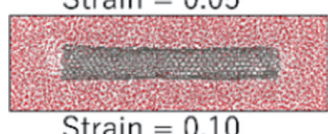

Strain $=0.10$

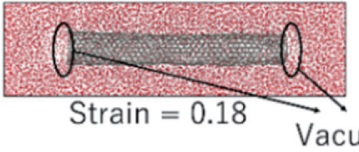

Vacuums (b)

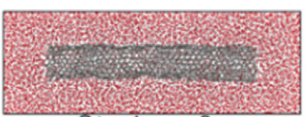
Strain $=0$

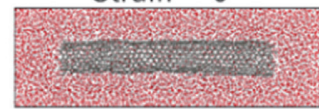

Strain $=0.05$

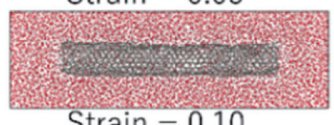
Strain $=0.10$

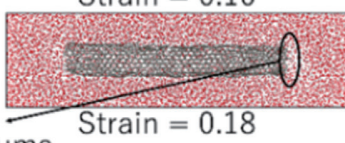

Figure 3. Simulation snapshots of the (a) non-annealed and (b) annealed alumina/CNT composites.

and $8.7 \mathrm{GPa}$, respectively) are higher than those of alumina (67.0 and 7.2 GPa, respectively). This result is consistent with the general expectation that CNTs reinforce the alumina matrix. Furthermore, the annealed composite shows higher Young's modulus and tensile strength (142.9 and 10.5 GPa, respectively) than the non-annealed composite, indicating the reinforcement by annealing.

To understand the reinforcing mechanism, we examine the snapshots of two alumina/CNT composites in tensile simulation, as shown in Figure 3. The snapshots before tensile (strain $=0)$, during elastic deformation ( $\operatorname{strain}=0.05$ ), and during plastic deformation (strain $=0.10$ and strain $=0.18$, corresponding to the points before and after tensile strength point, respectively) are taken to exhibit different deformation status. As shown in Figure 3a, in the non-annealed composite, 


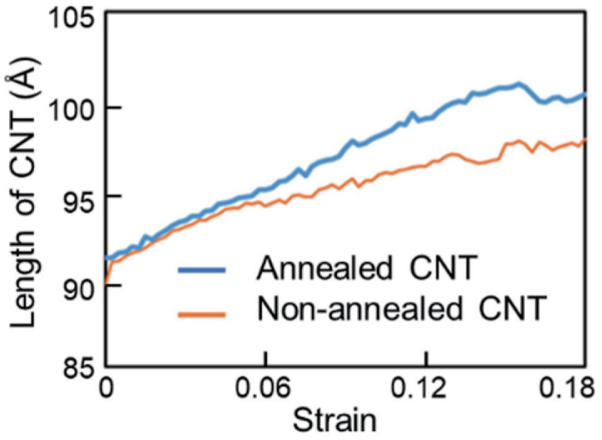

Figure 4. Length of CNT in the non-annealed and annealed composites during the tensile simulation.

vacuum areas appear at both ends of CNT at the strain of 0.18 , indicating the slippage at alumina/CNT interface and hence the CNT is not stretched as much as the alumina matrix. Meanwhile, in the annealed composite, the vacuum area mainly appears at the right end of CNT at the strain of 0.18 (Figure 3b), indicating that interfacial slippage is reduced by annealing. Thus, the annealed CNT is expected to be more stretched than the non-annealed CNT. To quantitatively confirm the stretch of CNT, the time evolution of the lengths of the non-annealed and annealed CNTs are surveyed, as shown in Figure 4. Obviously, although the initial lengths of both CNTs are almost the same, the length of the annealed CNT becomes larger than that of the non-annealed one during the tensile simulation of composites. This proves that $\mathrm{CNT}$ in the annealed composite is more stretched than the one in the non-annealed composite.

Why is the annealed CNT more stretched than the nonannealed one during the tensile simulation? In fact, the tensile stress is applied on the alumina matrix rather than the CNT, while the CNT is stretched only via the interfacial interactions between CNT and alumina. Thus, the different stretching behaviors of the annealed and non-annealed CNT maybe stem from the interfacial interactions. To evaluate the interfacial interactions, the number of the interfacial bonds $(\mathrm{C}-\mathrm{O}$ and $\mathrm{C}-\mathrm{Al}$ ) between the CNT and alumina is investigated. Since the bond-order-based potential of ReaxFF is employed, we are able to simply use a cutoff of bond order [6] to calculate the number of bonds. In this work, two atoms are recognized to be bonded when the bond orders of them are higher than the cutoff of 0.7 . For the non-annealed composite, the total number of interfacial bonds averaged over the last $10 \mathrm{ps}$ is 28.8 . Among these bonds, there are 15.0 bonds at the cylindrical region, 8.0 bonds at the left end, and 5.8 bonds at the right end of $\mathrm{CNT}$, indicating that the cylindrical region of CNT contributes more than the end sides to the overall interfacial interactions. For the annealed composite, the total number of interfacial bonds is 35.2 , showing an apparent increase contributed by annealing. This result verifies that the annealing could indeed improve interfacial interactions between CNT and alumina, so that the interfacial slippage is reduced by the strong interfacial interaction and hence the mechanical properties of CNT/ alumina composite are reinforced. Furthermore, in details, the number at the cylindrical region of annealed CNT is 24.2, while the numbers at the left and right end sides are 8.0 and 3.0, respectively, indicating that after annealing the interaction between alumina and the left end side of CNT becomes much stronger than that of the right end side. This result is also able to explain why vacuum is only generated at the right end side of annealed CNT at the end of the tensile simulation, as shown in Figure 3b. Overall, we conclude that annealing enhances the interfacial interactions between alumina and CNT by increasing the interfacial bonds so that the annealed CNT is more stretched than the non-annealed CNT, finally resulting in an improvement of the mechanical performance of composite.

\section{Conclusion}

In this study, the reinforcing mechanism of the alumina/ CNT composites from annealing is investigated by MD simulations. We find that the annealed alumina/CNT composite shows better Young's modulus and tensile strength than the non-annealed composite. This is because annealing enhances the interfacial interactions between the alumina and CNT and reduces the interfacial slippage, so that the annealed CNT is more stretched than the non-annealed CNT. Therefore, we reveal that the annealing improves the performance of CNT as reinforcement agent by enhancing the interfacial interactions between $\mathrm{CNT}$ and the matrix. 


\section{Reference}

[1] G. Yamamoto, K. Shirasu, Y. Nozaka, W. Wang, T. Hashida, Mater. Sci. Eng. A, 617, 179 (2014). DOI:10.1016/j.msea.2014.08.068

[2] K. Shirasu, G. Yamamoto, Y. Nozaka, W. Wang, T. Hashida, J. Mater. Sci., 50, 6688 (2015). DOI:10.1007/ s10853-015-9223-6

[3] J. Xu, Y. Higuchi, N. Ozawa, K. Sato, T. Hashida, M. Kubo, ACS Appl. Mater. Interfaces, 9, 31816 (2017). DOI:10.1021/acsami.7b07737, PMID:28849652
[4] S. G. Srinivasan, A. C. T. van Duin, P. Ganesh, J. Phys. Chem. A, 119, 571 (2015). DOI:10.1021/jp510274e, PMID:25562718

[5] S. Hong, A. C. T. van Duin, J. Phys. Chem. C, 120, 9464 (2016). DOI:10.1021/acs.jpcc.6b00786

[6] A. C. T. van Duin, S. Dasgupta, F. Lorant, W. A. Goddard, J. Phys. Chem. A, 105, 9396 (2001). DOI:10.1021/jp004368u 